\title{
Estrategias Cognitivas de Aprendizaje y Estrategias de Control en el Estudio y su relación con el rendimiento académico en estudiantes de Psicología de la Universidad de La Laguna
}

\section{Cognitive Learning Strategies and Study Control Strategies and their relationship with academic performance in Psychology students at the University of La Laguna}

\author{
$M^{\mathrm{a}}$ Isaura Felipe Afonso ${ }^{1}$, Luis A. García ${ }^{2}$, José Juan Castro Sánchez ${ }^{3}$ \\ ${ }^{1}$ Universidad de La Laguna misaurafa@gmail.com \\ ${ }^{2}$ Universidad de La Laguna lagarcia@ull.edu.es \\ ${ }^{3}$ Universidad de Las Palmas de Gran Canaria josejuan.castro@ulpgc.es
}

Recibido: 4/9/2020

Aceptado: 16/12/2020

Copyright (C)

Facultad de CC. de la Educación y Deporte.

Universidad de Vigo

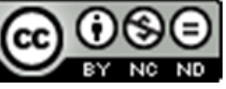

Dirección de contacto: $\mathrm{M}^{\mathrm{a}}$ Isaura Felipe Afonso Camino Las Camelias 48 38260 Tejina - La Laguna Santa Cruz de Tenerife

Canarias - España

\begin{abstract}
Resumen
Basándonos en estudios anteriores sobre las variables que influyen en el rendimiento académico universitario, presentamos como objeto de esta investigación, el estudio de la relación entre las estrategias cognitivas de aprendizaje y del control del estudio con el rendimiento académico de la población universitaria. Se ha realizado un estudio experimental descriptivo con 764 estudiantes pertenecientes a grupos que cursaban $3^{\circ}$ de Psicología desde el año 2013 hasta el año 2019. A través de las pruebas Estrategias Cognitivas de Aprendizaje (ECA) y Estrategias de Control en el Estudio (ECE), recogiendo a su vez, el rendimiento académico. Se llevó a cabo una prueba de diferencias de medias t-Student para grupos independientes según sus notas académicas, formándose así, un grupo denominado BajoR (33\% de alumnos/as con puntuaciones inferiores a 6 sobre 10$)$ frente a AltoR $(33 \%$ de alumnos/as con puntuaciones superiores a 7,5 sobre 10). Los resultados muestran que los estudiantes con mejor rendimiento, utilizan más estrategias de estructuración y elaboración del contenido y nemotecnias. Sin embargo, los estudiantes con peores resultados, utilizan como estrategias el aprendizaje receptivo (aprender el contenido tal y como aparece), el aprendizaje mecánico-superficial (sin entender) y el aprendizaje repetitivo. A su vez, los estudiantes con mejor rendimiento utilizan más estrategias de control del estudio que los estudiantes con peor rendimiento, encontrando diferencias significativas en las estrategias como revisión, planificación, búsqueda de alternativas, control del tiempo, reaprendizaje, detallar las tareas, controlar las variables externas y contar con apoyos personales para el estudio. Estos datos nos muestran la necesidad de valorar la mejora del rendimiento académico universitario a través de la aplicación estrategias de aprendizaje óptimas y de unas adecuadas técnicas de control del estudio.
\end{abstract}

Palabras clave

Estrategias, Control, Aprendizaje, Rendimiento. 


\begin{abstract}
Based on previous studies about factors that influence at university academic performance, we present as an object of this research, a study of the relationship between cognitive learning strategies and study control with the academic performance of the university population. A descriptive experimental study has been carried out with 764 students belonging to groups of 3rd year of Psychology from 2013 to 2019, through the Cognitive Learning Strategies (ECA) and Control Strategies in the Study (ECE) tests, including academic performance. A mean difference Student's t-test was carried out for independent groups according to their academic grades, in consequence creating a group called LowR $(33 \%$ of students with scores below 6 out of 10) versus HighR (33\% of students with scores higher than 7.5 out of 10). The results show that the students with better performance use more strategies of structuring and elaboration of the content and mnemonics. However, the worst-performing students use receptive learning (learning the content as it appears), mechanical-superficial learning (without understanding) and repetitive learning as strategies. At the same time, the best performing students use more study control strategies than the worst-performing students, finding significant differences in strategies such as revision, planning, search for alternatives, time control, relearning, detailing tasks, controlling external variables and resort to personal support for the study. These results show us the need to assess the improvement of university academic performance through the application of optimal learning strategies and adequate study control techniques.
\end{abstract}

\title{
Key Words
}

Strategies, Control, Learning, Performance.

\section{INTRODUCCIÓN}

La trayectoria de la investigación sobre el complejo mundo del aprendizaje, se ha visto marcada fundamentalmente por la exploración de diferentes áreas de estudio y fusiones dentro de este campo. Se han alcanzado, como resultado, numerosos hallazgos a lo largo de la historia desarrollados por distinguidos investigadores desde diferentes partes del planeta.

Muchas han sido las investigaciones focalizadas en descubrir, desde diversas perspectivas, los mecanismos implicados en el proceso de aprendizaje y todas ellas apuntan que, entre las variables que intervienen o condicionan el aprendizaje, algunas son inherentes al estudiante y otras relacionadas con el contexto. Estudios pioneros como los de Marton y Säljö (1976) aportaron respuestas que sirvieron de punto de partida para futuras investigaciones (Duff y McKinstry, 2007).

En los años 80, uno de los hitos que destacaron en una época focalizada en la perspectiva cognitiva del aprendizaje, fue el Proyecto Odyssey desarrollado por Adams (1984) (Beltrán, Moraleda, García-Alcañiz, Calleja y Santiuste, 1995), además de las aportaciones de conocidos autores como Pintrich, demostrando mejoras en el rendimiento académico de los estudiantes gracias a la enseñanza de estrategias cognitivas de aprendizaje (McKeachie, Pintrich y Lin, 1985), Gilles Einstein, (McDaniel y Einstein, 2019), Stenberg (1988), Deci y Ryan (1985) con su Teoría de la Autodeterminación y la motivación intrínseca (Stover, Bruno, Uriel y Fernández, 2017) o Danserau (1978) y Weinstein (1982 y 1986) con su taxonomía de estrategias de 
aprendizaje (Meza, 2013). En España en esta época fueron significativos los hallazgos de autores como Carles Monereo (1985) o Juan Ignacio Pozo (1989).

En una segunda etapa, en los años 90, la investigación comenzó a centrarse en conocer los procesos y estrategias de aprendizaje implicados en el estudio de materias concretas como los idiomas, ciencia o arte, entre otras. Resaltando autores como McDaniel y Einstein (1990) o Howard Gardner, quien despuntó con sus estudios sobre las inteligencias múltiples. Por su parte en España, y siguiendo esta línea, destacaron en este campo investigadores como González Cabanach (Lozano, Cabanach, Núñez, Porto y Santórum, 1990), Esteban, Ruiz y Ramírez (1996) o Jesús Beltrán (Deleito, Beltrán y Cobeñas, 2009).

En los inicios del siglo XXI, las investigaciones profundizaron en la relevancia de las emociones como elemento de influencia en los procesos de aprendizaje de los estudiantes, corriente respaldada por los trabajos de Daniel Goleman en 1995. En los últimos tiempos hasta la actualidad, se ha investigado sobre la implicación de la motivación, con aportaciones como las de Alderman (2008) o Pintrich y Schunk (2002) y sobre la autorregulación en el proceso de aprendizaje, como las aportaciones de Zimmerman y Schunk, (2001). En nuestro país, González Cabanach, Valle (Valle, Cabanach et al., 2006), Luis A. García (Martin, García, Torbay y Rodríguez, 2008) o De la Fuente et al. (2008), realizaron importantes hallazgos en estas últimas tendencias.

Tal y como se puede observar, a lo largo de la historia de este campo de estudio, no se han producido investigaciones independientes o estancas, sino que se han ido añadiendo y fusionando nuevas tendencias al tema principal de estudio, el proceso de aprendizaje.

Recientes investigaciones han profundizado en el estudio de los factores y circunstancias que influyen en el rendimiento académico universitario. Dichos hallazgos han encontrado que los factores sociodemográficos como el nivel socioeconómico, la edad y/o el apoyo social, los hábitos individuales respecto a la salud física como el ejercicio físico, el consumo de drogas y/o la buena alimentación y por último, la salud mental como trastornos del estado de ánimo y/o problemas para dormir, han arrojado resultados significativos en cuanto a su relación y condicionamiento del rendimiento académico de los universitarios (Alhadabi y Karpinski, 2020).

Esta realidad, sumada al mito desmontado de que un estudiante universitario tiene que estar capacitado para afrontar sus estudios con éxito, por el simple hecho de ser universitario/a (Martín et al., 2008), nos ha motivado a considerar que nuestra investigación y desde nuestro campo de actuación, debía de centrarse en las estrategias cognitivas de aprendizaje utilizadas por el alumnado universitario, así como el control ejercido en el estudio. Este trabajo parte de los estudios realizados en 2008 por Martin, García, Torbay y Rodríguez sobre las estrategias de aprendizaje universitario y su relación con el rendimiento académico.

Somos conscientes de que existen estudios que confirman que entre los factores que influyen en el rendimiento universitario, diferenciándose entre contextuales e individuales, estos últimos impactan en menor medida en el resultado académico (Alhadabi y Karpinski, 2020). Según Robbins et al., (2004) los factores cognitivos interpretados como individuales afectan en un $25 \%$ del rendimiento académico alcanzado.

En la actualidad, encontramos interesantes hallazgos sobre las estrategias cognitivas de aprendizaje, como las aportaciones de Trelles, Alvarado y Montánchez (2018), con 
sus estudios desarrollados a partir de una muestra de estudiantes de psicología educativa. Estos autores afirman que las estrategias de aprendizaje del alumnado universitario están relacionadas estrechamente con sus preferencias y necesidades, sugiriendo la necesaria intervención con dicha población, en aras de mejorar su pensamiento metacognitivo y fomentar su autonomía en la construcción de su propio aprendizaje. Además concluyen que las estrategias de aprendizaje utilizadas por los alumnos/as, contribuyen significativamente con la adecuada y precisa adquisición de conocimientos, mejorando la codificación, memorización o la correcta selección de fuentes de información, entre otros. Asimismo, recomiendan el fomento de la enseñanza de estrategias de aprendizaje al alumnado universitario de forma sincronizada con el desarrollo de la personalidad de los mismos, para lograr con ello, una mejora en sus logros académicos.

Por su parte, investigaciones enfocadas en el proceso de autorregulación del estudio de los estudiantes universitarios, según sus motivaciones, emociones y estrategias de aprendizaje (Hendrie Kupczyszyn y Bastacini, 2019), señalan el uso de estrategias cognitivas como factor clave en la consecución de logros académicos. Estas consideraciones surgen, gracias a la flexibilidad de la que disponen los estudiantes a la hora de elegir de forma óptima entre distintas estrategias para el desarrollo adecuado de una tarea específica.

Coincidimos con las investigaciones de Adame, Rigo y Aguilar (2013), en cuanto a la estrecha influencia de los aspectos cognitivos y de motivación sobre el proceso de aprendizaje. Manifiestan que para poder aprender es necesario "saber cómo hacerlo, poder hacerlo, disponer de las capacidades, conocimientos, estrategias y destrezas (componentes cognitivos) y querer hacerlo, tener la disposición, intención y motivación suficientes (componentes de motivación) para conseguir las metas propuestas" (2013, p. 15). Por lo tanto, el no manejar las estrategias, ni saber cuándo y cómo utilizarlas (control en el estudio) llevará al alumnado a desmotivarse y fracasar en la consecución de sus objetivos (Núñez y González-Pumariega, 1996).

Monereo (2020) en una de sus últimas publicaciones, mantiene que el objetivo final de todo docente se concentra en conseguir que sus alumnos/as sigan aprendiendo de forma autónoma una vez finalicen sus estudios universitarios. Este autor considera que el aprendizaje autónomo requiere que el alumno/a tome decisiones independientes y razonables, convirtiéndose en un "aprendiz estratégico" cuando decide según el contenido que hay que adquirir, cómo y cuándo aprender. Estas conclusiones refuerzan la importancia que presentan las estrategias cognitivas y de motivación de aprendizaje, así como el control en el propio estudio, para poder alcanzar las metas académicas propuestas y, por ende, un óptimo rendimiento académico.

En cuanto al rendimiento académico objetivo, observamos estudios como los de Hendrie Kupczyszyn y Bastacini (2019), quienes afirman que aquellos alumnos/as que consiguen un profundo procesamiento de la información mediante la adquisición, codificación y almacenamiento obtienen un rendimiento académico superior (Schmeck y Grove, 1979; Tomas y Rohwen 1986; Nisbet y Shucksmith, 1987). A su vez, Esguerra y Guerrero (2010) en sus trabajos con estudiantes de Psicología, encontraron una importante relación entre el modo de aprender y el rendimiento académico de los estudiantes.

Otros estudios actuales, afirman encontrar una relación significativa entre los hábitos de estudio y el rendimiento académico, sin embargo no encontraron la misma relación 
con la autoestima del alumnado (Chilca, 2017). Resultados similares se han encontrado en otras investigaciones, donde informan que aquellos estudiantes que alcanzan mejores calificaciones utilizan con mayor frecuencia estrategias de aprendizaje. Sin destacar la preferencia de unas estrategias sobre otras, sino más bien realizar una elección estratégica de las mismas, según el tipo de tarea que se tenga que afrontar (Juárez, Rodríguez, Escoto y Luna, 2016).

Incluso algunas investigaciones defienden que la competencia subjetiva que tenga el alumnado sobre sí mismo como estudiante, es una de las creencias de motivación que más influyen sobre su capacidad para desarrollar una tarea determinada y por lo tanto en su rendimiento académico objetivo (Boekaerts, 2002; Fast et al. 2010; Marcou y Philippou, 2005). Además, coinciden en que la competencia subjetiva es un factor importante en el aprendizaje autorregulado, ya que influye en las expectativas que tiene el alumno de poder desarrollar la tarea eficazmente (Musso, 2016; Hendrie Kupczyszyn y Bastacini, 2019).

Estudios, como los realizados por Navea y Varela (2019), consideran la autoeficacia percibida por los estudiantes universitarios sobre su propio rendimiento, como la variable que más aporta para poder predecir el rendimiento objetivo de los mismos, añadiendo en un segundo lugar como predictores del éxito académico, la gestión del tiempo de estudio y el lugar (control en el estudio).

Estas contribuciones se asemejan a las aportadas por De Besa, Gil y García, (2019) quienes encontraron evidencias para poder confirmar que "cuanto más consciente es el individuo de que dispone de las habilidades necesarias para la realización optima de cualquier tarea, mayores serán sus expectativas de éxito" (p. 159), siendo coherentes con estudios previos que relacionaban positivamente optimismo con autoeficacia (Jovanovi y Gavrilov- Jerkovi, 2013; Morton, Mergler y Boman, 2014).

\section{MÉTODO}

\subsection{Muestra}

Este estudio se ha realizado con la participación de 764 alumnos y alumnas pertenecientes a grupos tanto de la mañana como de la tarde, que cursaban $3^{\circ}$ del Grado de Psicología en la Universidad de La Laguna desde la promoción de 2013/2014 hasta el curso académico 2018/2019. Los datos sociodemográficos que hemos considerado relevantes para la realización de este estudio se muestran a continuación.

Tal y como se puede observar en la Figura 1, la muestra está formada mayoritariamente por mujeres con un $78 \%$ de representación en la muestra, frente a los hombres con un $22 \%$ de participación.

\begin{tabular}{ccc}
\hline Sexo & $\mathrm{N}$ & $\%$ \\
\hline Mujeres & 594 & 78 \\
\hline Hombres & 170 & 22 \\
\hline
\end{tabular}

Tabla 1. Valores y porcentajes de la muestra según su sexo

En cuanto a la edad de los alumnos y alumnas participantes, mostrada en la Figura 2, encontramos 3 edades principales, siendo el $46 \%$ de los participantes de 20 años, dato 
coherente con el estudio realizado e informando que casi la mitad de los participantes van cursando año por año. El 22\% son hombres y mujeres de 21 años y el 11\% tenían 22 años, agrupando así casi en el 80\% de la muestra, edades comprendidas entre 20 y 22 años.

\begin{tabular}{ccc}
\hline $\begin{array}{c}\text { Edades /Rango } \\
\text { Edades }\end{array}$ & $\mathrm{N}$ & $\%$ \\
\hline 20 años & 351 & 46 \\
\hline 21 años & 168 & 22 \\
\hline 22 años & 84 & 11 \\
\hline $23-28$ & 130 & 17 \\
\hline $29-55$ & 31 & 4 \\
\hline
\end{tabular}

Tabla 2. Valores y porcentajes de la muestra según edades

\subsection{Instrumentos}

Los instrumentos utilizados para realizar el presente estudio fueron los siguientes.

Cuestionario sobre Estrategias Cognitivas de Aprendizaje (ECA) (Hernández y García, 1991). Esta prueba está compuesta por un total de 44 ítems clasificados en 11 factores: Estructuración, Elaboración, Esencialización, Aprendizaje Receptivo, Comprensión Elaborada, Aprendizaje Mecánico-Superficial, Voz Alta, Nemotecnia, Aprendizaje Maximalista, Aprendizaje Repetitivo, Aprendizaje Cooperativo y Oral. La forma de respuesta de esta prueba es de tipo Likert ( $1=$ No lo hago nunca, $2=$ Lo hago pocas veces, $3=$ Lo hago algunas veces, $4=$ Lo hago bastantes veces y $5=$ Lo hago siempre); se solicita al participante que indique su nivel de acuerdo con lo que indica cada ítem, según la frecuencia con la que utiliza las distintas actividades, estrategias o modos de estudio para el aprendizaje en su titulación universitaria, indicándolo con una puntuación del 1 al 5.

Cuestionario sobre Estrategias de Control en el Estudio (ECE) (Hernández y García, 1991). Esta prueba está compuesta por un total de 17 ítems clasificados en 8 factores: Revisión, Planificación, Control del Tiempo, Buscar Alternativas, Reaprendizaje, Detalle Tarea, Variable-Apoyo, Función Examen. La forma de respuesta es de tipo Likert ( $1=$ No lo hago nunca, $2=$ Lo hago pocas veces, $3=$ Lo hago algunas veces, $4=$ Lo hago bastantes veces y $5=$ Lo hago siempre); se solicita al participante que indique su nivel de acuerdo con lo que indica cada ítem, según las estrategias que utilice tanto al inicio del estudio, como durante el estudio y al finalizar de estudiar, indicándolo con una puntuación del 1 al 5.

En todas las pruebas, el alumnado fue informado de que los datos obtenidos serian tratados con absoluta confidencialidad y solo se manejarían resultados globales. De esta forma, se les alentó para que contestasen con sinceridad y con ello colaborasen con la investigación.

\subsection{Procedimiento}

Se procedió a crear un cuestionario online utilizando la plataforma de formularios de Google, incorporando en él los instrumentos citados anteriormente. Se consiguió 
hacerlo llegar a todos los alumnos y alumnas a través de un enlace online que les permitía acceder al formulario, disponible en el campus virtual de la Universidad de La Laguna para los estudiantes matriculados en la asignatura "Evaluación e Intervención Psicoeducativa" correspondiente al tercer curso de la titulación de Psicología de la Universidad de La Laguna.

Consideramos fundamental destacar que el profesor universitario encargado de impartir dicha asignatura, ha sido el mismo docente en todos los cursos académicos en los que se han recopilado los datos para el presente estudio. Por lo tanto, se han utilizado las mismas estrategias metodológicas y los mismos criterios de evaluación para todo el alumnado. Este hecho es evidente y queda reflejado en el porcentaje de éxito y fracaso obtenido en esta asignatura en cada curso académico, el cual se ha mantenido constante a lo largo del tiempo.

\section{ANÁLISIS DE LOS DATOS}

Los datos han sido analizados a través del programa estadístico "Statistical Package for the Social Sciences" (SPSS), versión 25.

Para poder contrastar las hipótesis planteadas en el presente estudio se decidió crear tres grupos de alumnos y alumnas según la nota obtenida en la asignatura, el Grupo 1 compuesto por los datos de aquellos que obtuvieron las calificaciones más bajas (De 0 a 5,9) con $\mathrm{N}=266$; el Grupo 2 formado por el alumnado con notas medias (De 6 a 7,5) con $\mathrm{N}=261$; y el Grupo 3 compuesto por el alumnado que obtuvo las calificaciones más altas del curso (De 7,6 a 10 o Matrícula de Honor) con $\mathrm{N}=237$.

Una vez clasificado el alumnado según su calificación, se aplicó una prueba de diferencias de medias $\mathrm{t}$-Student para grupos independientes sobre la variable rendimiento académico, utilizando dos de los tres grupos creados, un grupo denominado BajoR (33\% de alumnos y alumnas con puntuaciones inferiores a 6 sobre 10) frente a otro grupo denominado AltoR (33\% de alumnos/as con puntuaciones superiores a 7,5 sobre 10). Con este análisis de datos se pretende hallar aquellos estilos de aprendizaje y estilos de pensamiento más frecuentes en cada uno de los grupos que se estudian y comprobar la existencia de diferencias significativas entre ambos.

\section{RESULTADOS}

Una vez realizado el análisis de datos y haciendo referencia a las estrategias cognitivas de aprendizaje utilizadas por los estudiantes universitarios, podemos comprobar en la Tabla 3 que el alumnado con un mayor rendimiento utiliza más estrategias de Estructuración que aquel con un rendimiento inferior $(\mathrm{t}=-2,38 ; \mathrm{p}<, 05)$. Se obtuvieron resultados similares para las estrategias de Elaboración $(\mathrm{t}=-2,83 ; \mathrm{p}<, 01)$ y Nemotecnia $(\mathrm{t}=-2,21 ; \mathrm{p}<, 05)$. Por otro lado, las estrategias cognitivas de aprendizaje más utilizadas por los estudiantes que obtuvieron las calificaciones más bajas fueron estrategias de Aprendizaje Receptivo ( $\mathrm{t}=4,41 ; \mathrm{p}<, 000)$, Aprendizaje Mecánico Superficial $(\mathrm{t}=4,55 ; \mathrm{p}<, 000)$ y Aprendizaje Repetitivo $(\mathrm{t}=3,89 ; \mathrm{p}<, 000)$. 


\begin{tabular}{ccccccc}
\hline Factor & $\begin{array}{c}\overline{\boldsymbol{x}}_{1} \\
\text { BajoR }\end{array}$ & $\mathrm{DT}$ & $\begin{array}{c}\overline{\boldsymbol{x}}_{2} \\
\text { AltoR }\end{array}$ & $\mathrm{DT}$ & $\mathrm{t}(\mathrm{gl})$ & $\mathrm{P}$ \\
\hline Estructuración & 3,35 & 0,72 & 3,50 & 0,72 & $-2,38(495,55)$ &, $018^{*}$ \\
\hline Elaboración & 3,47 & 0,75 & 3,66 & 0,76 & $-2,83(492,46)$ &, $005^{* *}$ \\
\hline Esencialización & 3,69 & 0,62 & 3,76 & 0,61 & $-1,30(496,56)$ & 0,195 \\
\hline $\begin{array}{c}\text { Aprendizaje } \\
\text { Receptivo }\end{array}$ & 2,37 & 0,78 & 2,07 & 0,73 & $4,41(499,27)$ &, $000^{* * *}$ \\
\hline $\begin{array}{c}\text { Comprensión } \\
\text { Elaborada }\end{array}$ & 2,45 & 0,46 & 2,53 & 0,50 & $-1,84(483,33)$ & 0,67 \\
\hline $\begin{array}{c}\text { Aprendizaje } \\
\text { Mecánico - } \\
\text { Superficial }\end{array}$ & 2,50 & 0,74 & 2,19 & 0,68 & $4,55(500,50)$ &, $000^{* * *}$ \\
\hline Voz Alta & 3,37 & 0,97 & 3,32 & 1,11 & $0,55(472,30)$ &, 580 \\
\hline Nemotecnia & 3,47 & 1,05 & 3,67 & 1,01 & $-2,21(498,06)$ & $0,027^{*}$ \\
\hline $\begin{array}{c}\text { Aprendizaje } \\
\text { Maximalista }\end{array}$ & 2,37 & 0,61 & 2,37 & 0,63 & $0,01(491,85)$ &, 991 \\
\hline $\begin{array}{c}\text { Aprendizaje } \\
\text { Repetitivo }\end{array}$ & 3,04 & 0,87 & 2,72 & 0,97 & $3,89(478,77)$ &, $000^{* * *}$ \\
\hline $\begin{array}{c}\text { Aprendizaje } \\
\text { Cooperativo y } \\
\text { Oral }\end{array}$ & 3,01 & 1,00 & 3,07 & 1,06 & $-0,58(486,99)$ &, 560 \\
\hline & & & & & & \\
\hline
\end{tabular}

Tabla 3. Diferencias de medias $\mathrm{t}$ - Student para muestras independientes (ECA)

Por otra parte, en cuanto a las estrategias de control en el estudio utilizadas por el alumnado universitario participante en la presente investigación, podemos observar en la Tabla 4, que el alumnado con mayor rendimiento académico utiliza, en general, más estrategias de control del estudio que los estudiantes con las calificaciones más bajas. Los resultados arrojaron diferencias significativas en este sentido, específicamente para las estrategias de Revisión ( $\mathrm{t}=-6,14 ; \mathrm{p}<, 000)$, Planificación $(\mathrm{t}=-5,09 ; \mathrm{p}<, 000)$, Control del Tiempo $(\mathrm{t}=-6,14 ; \mathrm{p}<, 000)$, Buscar Alternativas $(\mathrm{t}=-3,62 ; \mathrm{p}<, 000)$, Reaprendizaje $(\mathrm{t}=-4,46 ; \mathrm{p}<, 000)$, Detalle Tarea $(\mathrm{t}=-5,61 ; \mathrm{p}<, 000)$ y Control Variable - Apoyo $(\mathrm{t}=$ $4,49 ; \mathrm{p}<, 000)$.

\begin{tabular}{ccccccc} 
Factor & $\begin{array}{c}\overline{\boldsymbol{x}}_{1} \\
\text { BajoR }\end{array}$ & DT & $\begin{array}{c}\overline{\boldsymbol{x}}_{2} \\
\text { AltoR }\end{array}$ & $\mathrm{DT}$ & $\mathrm{t}(\mathrm{gl})$ & $\mathrm{P}$ \\
\hline Revisión & 3,67 & 0,71 & 4,05 & 0,67 & $-6,14(500,05)$ &, $000^{* * *}$ \\
\hline Planificación & 3,69 & 0,85 & 4,11 & 0,70 & $-6,13(498,85)$ &, $000^{* * *}$ \\
\hline $\begin{array}{c}\text { Control del } \\
\text { Tiempo }\end{array}$ & 3,39 & 1,28 & 3,95 & 1,20 & $-5,09(499,86)$ &, $000^{* * *}$ \\
\hline $\begin{array}{c}\text { Buscar } \\
\text { Alternativas }\end{array}$ & 9,84 & 1,60 & 10,37 & 1,66 & $-3,62(489,93)$ &, $000^{* * *}$ \\
\hline Reaprendizaje & 4,12 & 1,05 & 4,52 & 0,95 & $-4,46(500,79)$ &, $000^{* * *}$ \\
\hline Detalle Tarea & 15,09 & 3,71 & 16,77 & 3,02 & $-5,61(497,24)$ &, $000^{* * *}$ \\
\hline $\begin{array}{c}\text { Control } \\
\text { Variable - } \\
\text { Apoyo }\end{array}$ & 15 & 2,73 & 16,03 & 2,43 & $-4,49(500,99)$ &, $000^{* * *}$ \\
\hline $\begin{array}{c}\text { Función } \\
\text { Examen }\end{array}$ & 3,75 & 1,18 & 3,94 & 1,21 & $-1,73(491,31)$ &, 084 \\
\hline
\end{tabular}

Tabla 4. Diferencias de medias $\mathrm{t}$ - Student para muestras independientes (ECE) 


\section{DISCUSIÓN Y CONCLUSIONES}

Tal y como se aprecia en los resultados obtenidos, podemos observar que los estudiantes con mejor rendimiento académico hacen un uso más variado de estrategias cognitivas, sobre todo de aquellas que tienen que ver con una mayor elaboración y producción propia. En el caso de las estrategias de elaboración, estructuración y comprensión elaborada, requerirán del alumnado un mayor grado de pensamiento, ya que tiene que ver con habilidades cognitivas para relacionar y organizar la información y detectar lo relevante de la información aprendida.

En cambio, los estudiantes con un rendimiento más bajo se caracterizan por el uso de estrategias de aprendizaje más centrados en la repetición y un pensamiento más superficial, tal y como reflejan las variables aprendizaje receptivo, aprendizaje mecánico y aprendizaje repetitivo.

Por otro lado, la utilización de nemotecnias como estrategia de aprendizaje es más usada por los estudiantes de mejor rendimiento, quizás por el grado de elaboración que requiere para asociar el contenido o generar imágenes que ayuden a retener la información.

Estos resultados se encuentran en la línea de trabajos tales como los de TrellesAstudillo et al. (2018), Adame, Rigo, Aguilar et al. (2013) o Monereo (2020), que recogen la importancia de las estrategias cognitivas más elaborativas y significativas para la obtención de mejores resultados con el rendimiento académico en niveles no universitarios y universitarios.

La pregunta que se deduce de estos resultados es obvia ¿por qué no entrenamos a nuestros estudiantes en este tipo de estrategias? O bien, ¿por qué no favorecemos metodologías docentes que requieran o faciliten el uso de estas estrategias? Incluso, ¿qué hace que los estudiantes de bajo rendimiento no se planteen el uso de estrategias productivas? Sería interesante plantear, para próximos estudios y en la línea del presente trabajo, cuál es el motor principal que impulsa a los estudiantes a utilizar unas estrategias más complejas frente a otras superficiales, así como conocer en qué medida la comunidad educativa puede intervenir e influir en un cambio positivo y significativo, sobre las estrategias de aprendizaje utilizadas por los estudiantes a lo largo de su etapa universitaria.

Pero son sin duda las estrategias de control del estudio, las que más diferencian a los estudiantes de mejor rendimiento de los que obtienen peores resultados. El control del tiempo, las actividades de planificación (antes), supervisión (durante), revisión (después), búsqueda de alternativas ante los problemas y tener en cuenta las demandas del profesor en los exámenes son, sin duda, aspectos claves en la optimización del rendimiento, más incluso que el uso de estrategias cognitivas de elaboración.

Un estudiante elaborativo y autorregulado es sin duda el que tiene el perfil óptimo para alcanzar un alto rendimiento académico en la Universidad, por lo que todos los esfuerzos deben estar centrados en la búsqueda de apoyo para conseguir este objetivo.

Los trabajos de Trelles-Astudillo et al. (2018); Hendrie Kupczyszyn y Bastacini, (2019); Adame, Rigo, Aguilar et al. (2013); Núñez y González-Pumariega, (1996); Juárez et al., 2016; Musso, 2016; Navea y Varela (2019) y sobre todo las contribuciones del autor Carles Monereo (2020), aportan un importante respaldo a la idea de potenciar estudiantes productivos y autorregulados, como clave para el éxito académico. Incluso se relaciona con el éxito profesional posterior, puesto que las personas que conocen y 
confían en su capacidad y autoeficacia para llevar a cabo una tarea cualquiera, tienen una mayor expectativa de éxito (De Besa et al., 2019; Jovanovi y Gavrilov- Jerkovi, 2013; Morton et al., 2014) y por lo tanto, mayor motivación e iniciativa para conseguirlo.

Dada la relevancia y contenido de las conclusiones extraídas en el presente estudio, consideramos fundamental seguir profundizando en este ámbito y realizar futuras investigaciones mediante otros procedimientos para el análisis de datos, utilizando técnicas más complejas como el estudio de variables metacognitivas o la aplicación del modelo de ecuaciones estructurales, así como la revisión y mejora de los instrumentos utilizados para la recopilación de los datos.

\section{BIBILIOGRAFÍA}

Adame, M.T., Aguilar, E.M. Casero, A., Colom, J., De la Iglesia, B., Grases, G., Luca de Tena, C., Pérez, J.A., Rigo, E., Rodríguez, R.I., Rosselló, M.R. y Sureda, I. (2013). Estrategias de estudio y trabajo en estudiantes universitarios de la UIB (Informe de Recerca Núm. 72013). Institut de Recerca i Innovació Educativa. http://www.recercaeducativa.org/

Alderman, M.K. (2008). Motivation for achievement: Possibilities for teaching and learning. Nueva York: Taylor \& Francis.

Alhadabi, A. y Karpinski, A.C. (2020). Grit, self-efficacy, achievement orientation goals, and academic performance in University students. International Journal of Adolescence and Youth, 25(1), 519-535. https://doi.org/10.1080/02673843.2019.1679202

Beltrán, J., Moraleda, M., García-Alcañiz, E., Calleja, F. y Santiuste, V. (1995). Psicología de la educación. Madrid: Eudema.

Boekaerts, M. (2002). Motivation to learn. En D. Bhaskara Rao. (Ed.), Successful Schooling (pp. 101-120). New Delhi: Discovery Publishing House.

Chilca, M.L. (2017). Autoestima, habitos de estudio y rendimiento académico en estudiantes universitarios. Propósitos y Representaciones, 5(1), 71-99. https://dialnet.unirioja.es/servlet/articulo?codigo $=5904759$

De Besa, M.R., Gil, J. y García, A.J. (2019). Variables psicosociales y rendimiento académico asociados al optimismo en estudiantes universitarios españoles de nuevo ingreso. Acta Colombiana de Psicología, 22(1), 152-174. https://doi.org/10.14718/acp.2019.22.1.8

Deci, E.L. y Ryan, R.M. (1985). Intrinsic motivation and self-determination in human behavior. New York: Plenum Press

De la Fuente, J.A., Pichardo, M.C., Justicia, F. y Berbén, A. (2008). Enfoques de aprendizaje, autorregulación y rendimiento en tres universidades europeas. Psicothema, 20(4), 705-711.

Deleito, P.M., Beltrán, J.L. y Cobeñas, T.L. (2009). Perfil en estrategias de aprendizaje de estudiantes de alto rendimiento en lengua castellana y literatura. Faísca: revista de altas capacidades, 14(16), 49-75.

Duff, A. y McKinstry, S. (2007). Students' approaches to learning. Issues in accounting education, 22(2), 183-214.

Esguerra, G. y Guerrero, P. (2010). Estilos de aprendizaje y rendimiento académico en estudiantes de Psicología Diversitas. Perspectivas en Psicología, 6(1), 97-109.

Esteban, M., Ruiz, C. y Ramírez, F.C. (1996). Los estilos de aprendizaje y el rendimiento en Ciencias Sociales y en Ciencias de la Naturaleza en estudiantes de Secundaria. Anales de Psicología/Annals of Psychology, 12(2), 153-166.

Fast, L.A., Lewis, J.L., Bryant, M.J., Bocian, K.A., Cardullo, R.A., Rettig, M. y Hammond, K.A. (2010). Does math self-efficacy mediate the effect of the perceived classroom environment on standardized math test performance? Journal of Educational Psychology, 102(3), 729740. https://doi.org/10.1037/a0018863 
Hendrie Kupczyszyn, K.N. y Bastacini, M.D. (2019). Autorregulación en estudiantes universitarios: Estrategias de aprendizaje, motivación y emociones. Revista Educación, 44(1), 327-344. https://doi.org/10.15517/revedu.v44i1.37713

Hernández, P. y García, L.A. (1991). Psicología y enseñanza del estudio. Madrid: Pirámide.

Jovanovi, V. y Gavrilov-Jerkovi , V. (2013). Dimensionality and validity of the serbian version of the Life Orientation Test-Revised in a sample of youths. Journal of Happiness Studies, 14(3), 771-782. doi: 10.1007/s10902-012-9354-2

Juárez, C., Rodríguez, G., Escoto, M. y Luna, E. (2016). Relación de los estilos y estrategias de aprendizaje con el rendimiento académico en estudiantes universitarios. Revista de Estilos de Aprendizaje, 9(17), 268-288.

Lozano, A.B., Cabanach, R.G., Núñez, J.C., Porto, A.M. y Santórum, R. (1990). La estructura cognitiva de los niños bilingües y no bilingües: Un estudio diferencial. Revista de Psicología General y Aplicada, 43(1), 97-104.

Marcou, A. y Philippou, G. (2005). Motivational beliefs, self-regulated learning and mathematical problem solving. En H.L. Chick y J.L. Vincent (Ed.), Proceedings of the 29th Conference of the International Group for the Psychology of Mathematics Education, (pp. 297-304). Melbourne: PME.

Martín, E., García, L.A., Torbay, Á. y Rodríguez, T. (2008). Estrategias de aprendizaje y rendimiento académico en estudiantes universitarios. International Journal of Psychology and Psychological Therapy, 8(3), 401-412.

Marton, F. y Säljö, R. (1976). On qualitative differences in learning I - outcomes and processes. British Journal of Educational Psychology, 46, 4-11.

McDaniel, M.A. y Einstein, G.O. (2019). Training Learning Strategies to Promote SelfRegulation and Transfer: The Knowledge, Belief, Commitment, and Planning Framework (submitted).

McKeachie, W.J., Pintrich, P.R. y Lin, Y.G. (1985). Teaching learning strategies. Educational Psychologist, 20(3), 153-160.

Meza, A. (2013). Estrategias de aprendizaje. Definiciones, clasificaciones e instrumentos de medición. Propósitos y representaciones, 1(2), 193-212.

Monereo, C.F. (1985). Los sistemas de orientación, consejo y asesoramiento en la integración escolar. Revista Educar, 8, 71-89.

Monereo, C.F. (2020). Enseñar y aprender en la educación superior. En M. Turull (Coord.), Manual de docencia universitaria (pp. 75-98). Barcelona: Ediciones Octaedro.

Morton, S., Mergler, A. y Boman, P. (2014). Managing the transition: The role of optimism and self-ef cacy for rst- year Australian university students. Australian Journal of Guidance and Counselling, 24(1), 90-108. doi:.10.1017/ jgc.2013.29

Musso, M.F. (2016). Understanding the underpinnings of academic performance: Students, asks and performance: A structural equations (SEM) approach to model the effect of cognitive and SRL factors in academic performance. (Doctoral thesis). KU Leuven: Bélgica.

Navea, A. y Varela, I. (2019). Variables motivacionales y cognitivas predictivas del rendimiento en estudiantes universitarios de ciencias de la salud. Educación Médica Superior, 33(1), 129.

http://scielo.sld.cu/scielo.php?pid=S086421412019000100007\&script=sci_abstract\&tlng= en

Nisbet, J. y Shucksmith. J. (1987). Learning Strategies. London: Routledge and Kegan Paul.

Núñez, J.C. y González-Pumariega, S. (1996). Procesos motivacionales y aprendizaje. Psicología de la Instrucción, 2, 33-64.

Pintrich, P.R. y Schunk, D.H. (2002). Motivation in education: Theory, research, and applications. Upper Saddle River, New Jersey: Prentice Hall.

Pozo, J.I. (1989). Teorías cognitivas del aprendizaje. Madrid: Ediciones Morata. 
Robbins, S., Lauver, K., Huy, L., Davis, D. y Langley, R. (2004). Do Psychosocial and Study Skill Factors Predict College Outcomes? A Meta-Analysis. Psychological Bulletin, 130(2), 261-288. doi: 10.1037/0033- 2909.130.2.261

Schmeck, R.R. y Grove, E. (1979). Academic achievement and individual differences in learning processes. Applied Psychological Measurement, 3(1), 43-49.

Sternberg, R.J. (1988). Mental self-government: A theory of intellectual styles and their development. Human Development, 31, 197-224.

Stover, J.B., Bruno, F.E., Uriel, F.E. y Fernández, M.L. (2017). Teoría de la Autodeterminación: una revisión teórica. Perspectivas en Psicología: Revista de Psicología y Ciencias Afines, 14 (2), 105-115.

Thomas, J.W. y Rohwer, W.D. (1986). Academic studying: The role of learning strategies. Educational psychologist, 21(1-2), 19-41.

Trelles, H.J., Alvarado, H.P. y Montánchez, M.L. (2018). Estrategias y estilos de aprendizaje y su relación con el rendimiento académico en estudiantes universitarios de Psicología Educativa. Killkana Social, 2(2), 9-16. https://doi.org/10.26871/killkana_social.v2i2.292

Valle, A., Cabanach, R.G., Rodríguez, S., Núñez, J. C. y González-Pienda, J.A. (2006). Metas académicas, estrategias cognitivas y estrategias de autorregulación del estudio. Psicothema, 18(2), 165-170.

Zimmerman, B.J. y Schunk, D.H. (Eds.). (2001). Self-regulated learning and academic achievement: Theoretical perspectives. (2nd Ed.) Mahwah, New Jersey: Lawrence Erlbaum. 